\title{
Note
}

\section{Complementation of the tomato HWS gene with its Arabidopsis counterpart demonstrates conservation of the gene function between both species}

\author{
Toshifumi Nagata ${ }^{1, \dagger}$, Fabien Lombardo ${ }^{1, \dagger}$, Hiroshi Ezura ${ }^{1,2, *}$ \\ ${ }^{1}$ Faculty of Life and Environmental Sciences, University of Tsukuba, 1-1-1 Tennodai, Tsukuba, Ibaraki 305-8572, Japan \\ ${ }^{2}$ Tsukuba Plant Innovation Research Center, University of Tsukuba, 1-1-1 Tennodai, Tsukuba, Ibaraki 305-8572, Japan \\ *E-mail: ezura.hiroshi.fa@u.tsukuba.ac.jp Tel \& Fax: +81-29-853-4796
}

Received June 5, 2021; accepted July 29, 2021 (Edited by R. Hayama)

\begin{abstract}
The HAWAIIAN SKIRT (HWS) gene was originally described in Arabidopsis for the characteristic fusion of sepals in the mutant. A tomato line mutated in the putative ortholog gene was isolated in a previous study. The tomato $h w s-1$ mutant showed facultative parthenocarpy and produced fruits with elevated Brix, revealing the gene as a hopeful resource for crop improvement. To confirm the orthology relationship between the Arabidopsis and tomato HWS genes, the hws- 1 mutant was complemented with either the tomato wild-type genomic fragment or the Arabidopsis sequence of the gene. In both complementation experiments, defective phenotypes of $h w s-1$ are rescued, albeit to different extents. Recovery of these phenotypes, which include parthenocarpic fruit production, increased Brix, loss of leaflet serration, alteration of bud and petal shape, firmly establishes SlHWS as an ortholog of the originally described HWS in Arabidopsis. This work indicates that the function of HWS is likely to be conserved in a wide range of plant species.
\end{abstract}

Key words: F-box gene, HAWAIIAN SKIRT, parthenocarpy, tomato.

Parthenocarpy can be defined as the production of plant fruits in the absence of ovule fertilization. The male reproductive function being particularly sensitive to heat stress (Peet et al. 1998), parthenocarpic crops are expected to be more resilient against unfavorably high temperatures with regards to fruit production. A tomato (Solanum lycopersicum) parthenocarpic line was previously isolated from a genetic screening of an ethyl-methanesulfonate-mutagenized population of the Micro-Tom cultivar (Saito et al. 2011; Shikata et al. 2016). The causal gene was identified as Solyc01g095370, which encodes an F-box protein showing more than $60 \%$ identical amino acids with the Arabidopsis (Arabidopsis thaliana) HAWAIIAN SKIRT (HWS) protein (Damayanti et al. 2019; González-Carranza et al. 2007). The mutant was therefore tentatively designated as slhws-1. In addition to the parthenocarpy trait, slhws-1 fruits showed an increased Brix level. As Brix level usually corresponds to sugar content, this finding further deepened the interest in the mutant in the context of crop improvement.

Although several pieces of evidence pointed towards the fact that both genes are orthologous (Damayanti et al. 2019), the phylogenetic distance between both species and the absence of fleshy fruits in Arabidopsis weakened any conclusions that could have been drawn from comparative phenotypic studies alone. To generate the lacking genetic evidence, two types of complementation experiments of the slhws-1 mutant were performed. In a first experiment, Solyc01g095370 is demonstrated to be the causal gene of the slhws-1 phenotype; a second experiment establishes SlHWS as the tomato ortholog of AtHWS. These are described in the following.

In a previous study, sequencing results in the tomato $h w s-1$ line revealed the presence of a cytosine-toadenosine transversion located towards the $3^{\prime}$ end of the CDS of the Solyc01g095370 gene (Damayanti et al. 2019). To firmly demonstrate that the mutation was responsible for the mutant phenotype, the full genomic sequence of Solyc01g095370 consisting of a 5,361 bp promoter and 1,218 bp CDS fragment (Figure 1A) was introduced into the slhws-1 following the standard transformation method (Sun et al. 2006). A minimum of nine transformants of the $\mathrm{T}_{2}$ generation, in parallel with a minimum of nine wild type (WT) individuals, was grown on rockwool blocks periodically supplied with Ohtsuka House 1 and 2 nutrient solutions (OAT Agrio Co., Ltd., Japan) in standard cultivation conditions $\left(22^{\circ} \mathrm{C}\right.$, under fluorescent lighting with a $16 \mathrm{~h} / 8 \mathrm{~h}$ light/dark cycle). All observations were done on 3-month-old plants with

${ }^{\dagger}$ These authors contributed equally to this work.

This article can be found at http://www.jspcmb.jp/

Published online September 18, 2021 
A

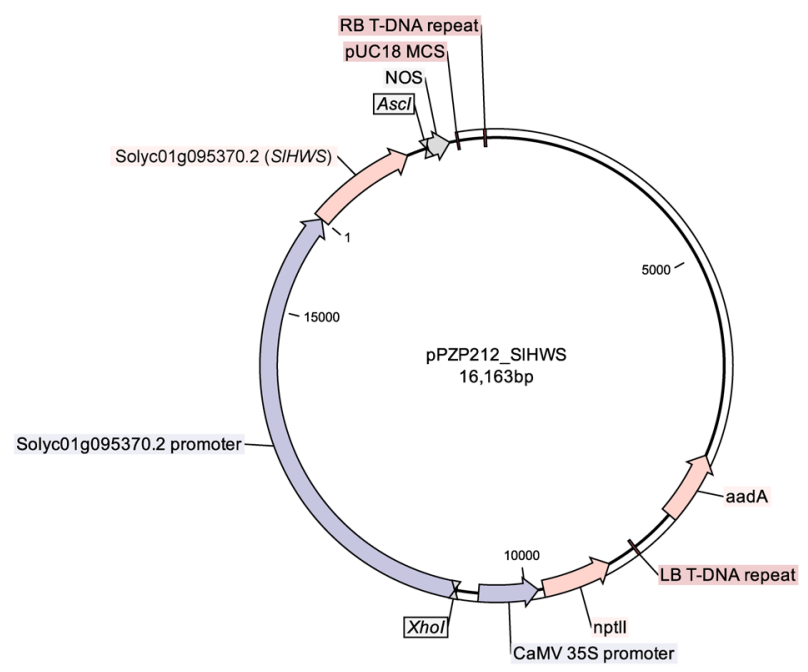

B

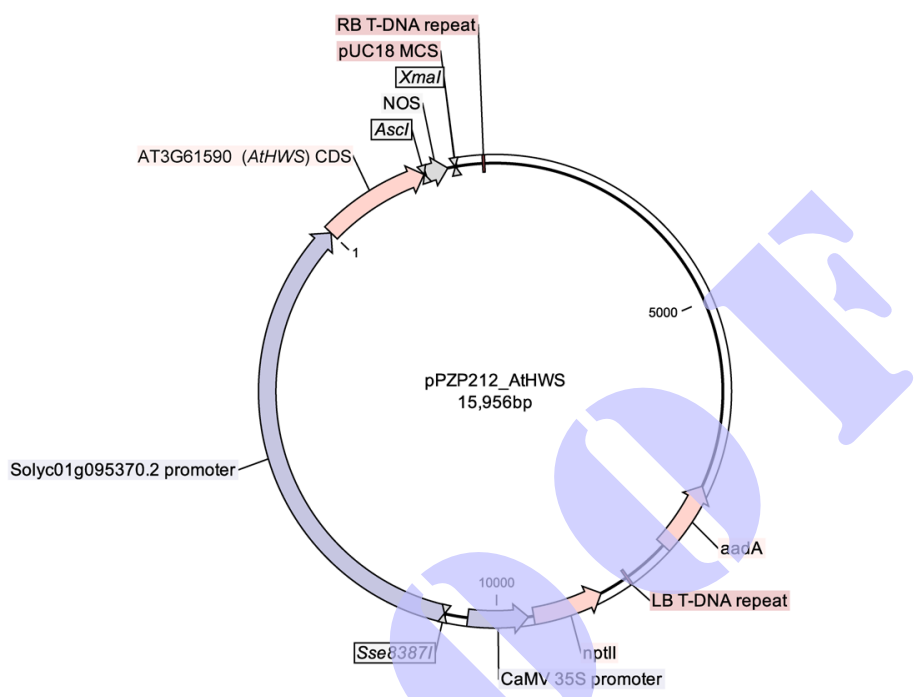

Figure 1. Design of transformation vectors. A. Full genomic fragment of the SlHWS gene in the pPZP212 vector backbone. B. AtHWS coding sequence fused to the tomato native promoter in the pPZP212 backbone.

A

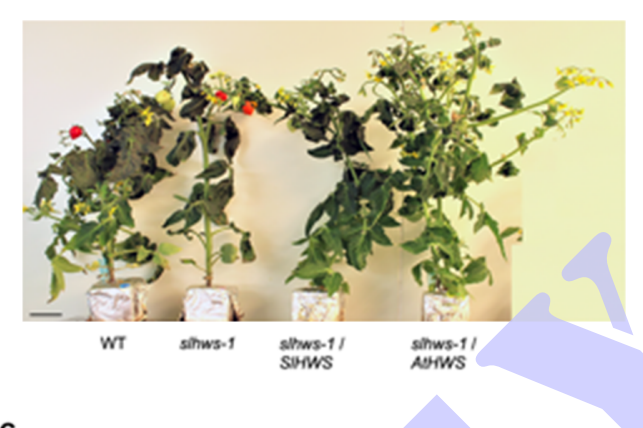

C

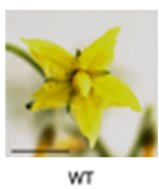

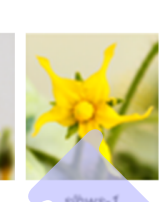

,

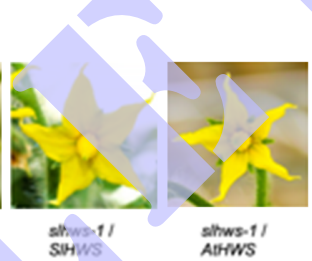

B
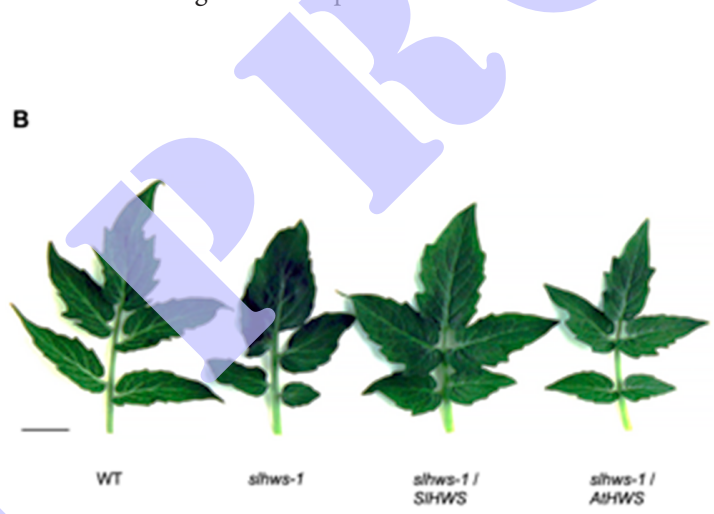

D
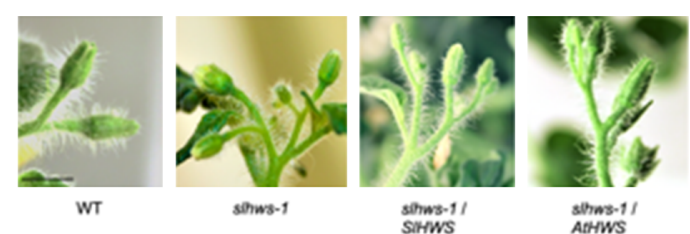

Figure 2. Representative visual features of the slhws-1 mutant compared with the WT control as well as with tomato and Arabidopsis complementation lines. A. Expanded overall plant architecture. Scale bar $=5 \mathrm{~cm}$. B. Reduction in leaflet serration. Scale bar $=2 \mathrm{~cm}$. C. Narrow-ended petal shape. Scale bar $=1 \mathrm{~cm}$. D. Barrel-shaped buds. Scale bars $=1 \mathrm{~cm}$.

fruits at the red ripe stage. Statistical significance in our different measurements was determined from an ANOVA test (type II, $p<0.05$ ) which was eventually followed by a Tukey's test.

In addition to the parthenocarpy and high sugar traits, several phenotypic features were associated with the presence of the mutation at the recessive state. Plant overall architecture was markedly altered in slhws-1, contrasting with the short and compact Micro-Tom WT. Mutants were about ten centimeters taller on average; the number of lateral shoots was halved, and stem diameter was also noticeably larger in $h w s-1$ (Figure 2A; Table 1). In all analyzed individuals complemented with the genomic fragment construct, these traits were partially rescued to the exception of the stem diameter, which was even smaller than the WT one. Brix was measured in triplicates using the pericarp of individual fruits after removal of seeds and jelly part using a refractometer PAL-J (Atago Co., Ltd., Tokyo, Japan). The fruits of hws-1 were of comparable diameter, however they were fewer and characterized by an elevated Brix (Table 1). Complementation with the WT genomic fragment allowed producing an intermediate number of fruits and completely restored WT Brix levels. Parthenocarpic fruit formation and seedless-fruit formation were counted from fruits more than $2 \mathrm{~g}$ of fresh weight and 
Table 1. Evaluation of the phenotypic rescue of $h w s-1$ in the complementation lines.

\begin{tabular}{lcccc}
\hline \multicolumn{1}{c}{ Trait } & WT & slhws-1 & slhws-1/SlHWS & slhws-1/AtHWS \\
\hline Plant height (cm) & $20.7 \pm 2.1^{\mathrm{b}}$ & $33.0 \pm 2.3^{\mathrm{a}}$ & $28.9 \pm 2.5^{\mathrm{ab}}$ & $28.3 \pm 1.5^{\mathrm{ab}}$ \\
Stem diameter (mm) & $4.91 \pm 0.13^{\mathrm{b}}$ & $5.56 \pm 0.10^{\mathrm{a}}$ & $3.98 \pm 0.09^{\mathrm{c}}$ & $4.75 \pm 0.18^{\mathrm{b}}$ \\
Lateral shoot number & $2.5 \pm 0.2^{\mathrm{a}}$ & $1.3 \pm 0.2^{\mathrm{b}}$ & $2 \pm 0.4^{\mathrm{ab}}$ & $2.2 \pm 0.1^{\mathrm{ab}}$ \\
Fruit number & $34.6 \pm 3.3^{\mathrm{a}}$ & $13.5 \pm 1.9^{\mathrm{b}}$ & $24 \pm 3.2^{\mathrm{ab}}$ & $18.4 \pm 2.8^{\mathrm{b}}$ \\
Fruit diameter (mm) & $19.3 \pm 1.2^{\mathrm{b}}$ & $17.4 \pm 1.2^{\mathrm{b}}$ & $15.9 \pm 0.6^{\mathrm{b}}$ & $17.0 \pm 0.4$ \\
Total soluble solids (Brix) & $4.8 \pm 0.1^{\mathrm{b}}$ & $8.6 \pm 0.6^{\mathrm{a}}$ & $5.8^{\mathrm{b}} \pm 0.4^{\mathrm{b}}$ & $5.2 \pm 0.2^{\mathrm{b}}$ \\
Ratio of seeded fruits (\%) & $100 \pm 0.0^{\mathrm{a}}$ & $7.5 \pm 5.4^{\mathrm{b}}$ & $83.3 \pm 8.3^{\mathrm{a}}$ & $90 \pm 10.0^{\mathrm{a}}$ \\
Seed number & $21.1 \pm 1.9^{\mathrm{a}}$ & $4.0 \pm 2.7^{\mathrm{b}}$ & $17.7 \pm 2.0^{\mathrm{a}}$ & $20.2 \pm 2.3^{\mathrm{a}}$ \\
\hline
\end{tabular}

Values represent mean + SE ( $n>9$ plants); statistically different groups as indicated with superscript letters; flowers were mechanical pollinated as in Damayanti et al. 2019.

the number of seeds was counted from 15 fruits in each line. The very low fertility associated with the formation of parthenocarpic fruits in slhws-1 was shown in an ealier study to result mainly from a defective male function (Damayanti et al. 2019). This trait was also rescued in the complemented lines (Table 1). Reduction in leaf serration has been associated with the $h w s$ phenotype in both Arabidopsis (Lang et al. 2018) and tomato (Damayanti et al. 2019). Serrations were restored on the leaflets of the slhws-1/SlHWS individuals, almost to the extent of the WT (Figure 2B). Similarly, the petals on the complemented individuals could barely be distinguished from the WT ones, whereas $h w s-1$ petals were strikingly narrow at their extremities (Figure 2C). Another distinctive feature of the mutant is the barrel shape of the flower buds associated with short sepals, leaving the tip of the anther cone uncovered (Figure 2D). Here again, complemented mutants with the genomic HWS fragment showed buds comparable to the WT ones. Collectively, these results indicate that Solyc01g095370 is the causal gene for the HWS phenotype.

To assess whether AtHWS could complement slhws-1, the CDS of SlHWS in the complementation construct described earlier was swapped with the 1,236-bplong CDS of AtHWS, leaving the rest of the vector unchanged (Figure 1B). Observation of a minimum of 12 transformants of the $T_{2}$ generation revealed that reproductive traits were either fully rescued or rescued to the same extent of the tomato WT genomic complementation, with the notable exception of fruit number, which was not significantly different from the mutant one (Table 1). Flower bud shape and leaflet serrations were barely distinguishable from the WT ones (Figure 2C, D). The overall plant aspect as well as the petal shape were intermediate between the mutant and the WT lines, indicating a partial rescue for these traits. Based on these results and results in the first complementation experiment data, it is concluded that the Arabidopsis HWS protein can rescue the slhws-1 phenotype, substantiating the claim that both genes are orthologs.

In rice, both the EP3 and FBK1 genes share a high homology with SlHWS (Damayanti et al. 2019;
Lombardo et al. 2021). The ep3 mutant shows an erect panicle shape, increase in the number of vascular bundles in specific regions of the panicle and an increase in grain yield ( $\mathrm{Li}$ et al. 2011; Piao et al. 2009). FBK1 has been described for its role in determining cell wall thickening by targeting for degradation a reductase involved in lignin synthesis (Borah and Khurana 2018). Although the functions of these genes do not seem to overlap, both EP3 and FBK1 can complement the Arabidopsis hws1 mutant (González-Carranza, unpublished; Yu et al. 2015). Interestingly, grain yield is increased in the ep3 mutant (Li et al. 2011). Most likely, the poplar PtaHWS is another ortholog (Dash et al. 2015), although to our knowledge there are no complementation experiments to confirm this hypothesis. In this work, complementation of the tomato mutant with the Arabidopsis sequence brings another piece of evidence supporting the idea that the gene function is remarkably conserved across unrelated species. It is expected that in a wide variety of plants, orthologs of HWS control aspects of fruit development. In tomato, Arabidopsis and poplar, functional disruption of the gene has been shown known to affect the microRNA metabolism and it is hypothesized that the mutant phenotypes are resulting from the accumulation of specific microRNAs species (Damayanti et al. 2019; Dash et al. 2015; Lang et al. 2018; Zhang et al. 2017). It would be interesting to confirm whether the microRNA metabolism is also altered in the rice mutants. Moreover, protein sequence analysis of the different orthologs would provide clues towards the identification of a putative target for HWS in the microRNA pathway.

\section{Acknowledgements}

T.N and F.L wrote the article and conducted the research. H.E supervised the research, provided resources, and acquired the funding. The authors want to thank the National BioResource Project of the University of Tsukuba, Japan for providing the tomato $h w s-1$ mutant line. This work was supported by JSPS KAKENHI Grant No. 25252008 and Grant No. 17H01461 to H.E., and Science and Technology Research Promotion Program for Agriculture, Forestry, Fisheries and Food Industry, Japan (Grant No. 26013A) to H.E. 


\section{References}

Borah P, Khurana JP (2018) The OsFBK1 E3 ligase subunit affects anther and root secondary cell wall thickenings by mediating turnover of a cinnamoyl-CoA reductase. Plant Physiol 176: $2148-2165$

Damayanti F, Lombardo F, Masuda J, Shinozaki Y, Ichino T, Hoshikawa K, Okabe Y, Wang N, Fukuda N, Ariizumi T, et al. (2019) Functional disruption of the tomato putative ortholog of HAWAIIAN SKIRT results in facultative parthenocarpy, reduced fertility and leaf morphological defects. Front Plant Sci 10: 1-25

Dash M, Yordanov YS, Georgieva T, Kumari S, Wei H, Busov V (2015) A systems biology approach identifies new regulators of poplar root development under low nitrogen. Plant J 1: 335-346

González-Carranza ZH, Rompa U, Peters JL, Bhatt AM, Wagstaff C, Stead AD, Roberts JA (2007) HAWAIIAN SKIRT: An F-box gene that regulates organ fusion and growth in Arabidopsis. Plant Physiol 144: 1370-1382

Lang PLM, Christie MD, Dogan ES, Schwab R, Hagmann J, van de Weyer A-L, Scacchi E, Weigel D (2018) A role for the F-box protein HAWAIIAN SKIRT in plant microRNA function. Plant Physiol 176: 730-741

Li M, Tang D, Wang K, Wu X, Lu L, Yu H, Gu M, Yan C, Cheng Z (2011) Mutations in the F-box gene LARGER PANICLE improve the panicle architecture and enhance the grain yield in rice. Plant Biotechnol J 9: 1002-1013

Lombardo F, Gramazio P, Ezura H (2021) Increase in phloem area in the tomato hawaiian skirt mutant is associated with enhanced sugar transport. Genes (Basel) 12: 932

Peet MM, Sato S, Gardner RG (1998) Comparing heat stress effects on male-fertile and male-sterile tomatoes. Plant Cell Environ 21: 225-231

Piao R, Jiang W, Ham T-H, Choi M-S, Qiao Y, Chu S-H, Park J-H, Woo M-O, Jin Z, An G, et al. (2009) Map-based cloning of the ERECT PANICLE 3 gene in rice. Theor Appl Genet 119: 1497-1506

Saito T, Ariizumi T, Okabe Y, Asamizu E, Hiwasa-Tanase K, Fukuda N, Mizoguchi T, Yamazaki Y, Aoki K, Ezura H (2011) TOMATOMA: A novel tomato mutant database distributing micro-tom mutant collections. Plant Cell Physiol 52: 283-296

Shikata M, Hoshikawa K, Ariizumi T, Fukuda N, Yamazaki Y, Ezura H (2016) TOMATOMA Update: Phenotypic and metabolite information in the Micro-Tom mutant resource. Plant Cell Physiol 57: e11

Sun HJ, Uchii S, Watanabe S, Ezura H (2006) A highly efficient transformation protocol for Micro-Tom, a model cultivar for tomato functional genomics. Plant Cell Physiol 47: 426-431

Yu H, Murchie EH, González-Carranza ZH, Pyke KA, Roberts JA (2015) Decreased photosynthesis in the erect panicle 3 (ep3) mutant of rice is associated with reduced stomatal conductance and attenuated guard cell development. J Exp Bot 66: 1543-1552

Zhang X, Jayaweera D, Peters JL, Szecsi J, Bendahmane M, Roberts JA, González-Carranza ZH (2017) The Arabidopsis thaliana F-box gene HAWAIIAN SKIRT is a new player in the microRNA pathway. PLoS One 12: $\mathrm{e} 0189788$ 